\title{
Homogenization of granular pipe flow by means of helical inner-wall texture
}

\author{
Felix Verbücheln ${ }^{1, \star}$, Eric J. R. Parteli ${ }^{2, \star \star}$, and Thorsten Pöschel ${ }^{1, \star \star \star}$ \\ ${ }^{1}$ University of Erlangen-Nuremberg, Erlangen, Germany \\ ${ }^{2}$ University of Cologne, Cologne, Germany
}

\begin{abstract}
The homogenization of granular flows through narrow pipes is important for a broad range of technological and industrial applications. Here we show, by means of molecular dynamics simulations, that such homogenization can be achieved by adding a helical inner-wall texture to the pipe, without the need for energy input from any external source. By using such a texture, jamming is prevented and the granular flux can be predicted using a modified Beverloo equation that accounts for the wavelength of the helical texture.
\end{abstract}

\section{Introduction}

Granular pipe flows are characterized by intermittent behavior and large, potentially destructive solid fraction variations in the transport direction. Although the dynamics of density waves in granular pipe flows have been studied extensively in the past [1-5], it is still a challenging problem to control the mass flux of the granular material flowing through a pipe (see e.g. Ref. [6]).

Most of the proposed strategies to control the transport along the pipe involve energy input from an external source, e.g. through application of electric fields [7] or mechanical perturbations [8]. One exception is the work of Zuriguel et al. [9], who showed how insertion of an obstacle just above the outlet of a silo can significantly reduce the probability that the granular flow is arrested due to the formation of an arch blocking the silo's outlet [9].

Here we demonstrate a method to homogeneize the mass flux and avoid flow blockage in granular pipe flows without necessity of applying any external source of energy to the system. By means of particle-based numerical experiments, we will show that it is possible to achieve flows with prescribed characteristics regarding the particle distribution within the pipe and the mass flow rate of the granular material by adjusting the geometric properties of the helix-shaped texture.

\section{Numerical experiments}

We simulate the process using the Discrete Element Method (DEM), that is, simultaneously solving Newton's equations of translational and rotational motion for all particles. We assume viscoelastic interaction in normal direction $[10,11]$ and apply a modified Cundall-Strack model [12] for the tangential direction [13]. The integration was

\footnotetext{
^e-mail: verbuechelnf@googlemail.com

$\star \star$ e-mail: eric.parteli@uni-koeln.de

$\star \star \star$ e-mail: thorsten.poeschel@fau.de
}

performed using LIGGGHTS [13, 14], while the values of the model parameters are listed in Tab. 1. While a detailed description of the model has been presented before [1418], in the following we focus on the description of the numerical experiments of granular pipe flow and, in the subsequent section, the model of the helix-shaped texture to homogenize the particle transport [14].

The pipe has a circular cross-section of diameter $D \mathrm{~mm}$ and its length is $L=1 \mathrm{~m}$. The inner-wall of the pipe is frictional, though it is modelled as a flat surface, i.e. the pipe is not built with particles (see Ref. [14]). At time $t=0$, $N$ particles are placed at random positions within the pipe, where $N$ is chosen such that the sum of the volumes of all particles amounts to a prescribed fraction $V_{\phi}$ of the pipe volume. The initial velocity of the particles in radial direction is chosen randomly between $-v_{r}$ and $v_{r}$, with $v_{r}=0.01$ $\mathrm{m} / \mathrm{s}$, while the particles have vanishing initial velocity in the axial direction $\left(v_{z}=0\right)$. Periodic boundary conditions are applied in the vertical $(z)$ direction.

Table 1. Numerical values of the parameters used in the simulations.

\begin{tabular}{lcl}
\hline parameter & symbol & value \\
\hline particle material density & $\rho_{\mathrm{p}}$ & $2650 \mathrm{~kg} / \mathrm{m}^{3}$ \\
particle diameter & $d$ & $1.2 \mathrm{~mm}$ \\
Young's modulus & $Y$ & $10^{8} \mathrm{~Pa}$ \\
Poisson's ratio & $v$ & 0.24 \\
Coulomb's friction coefficient & $\mu$ & 0.5 \\
pipe length & $L$ & $1 \mathrm{~m}$ \\
timestep & $\Delta t$ & $6 \times 10^{-7} \mathrm{~s}$ \\
\hline
\end{tabular}

\section{Results and Discussion}

We perform simulations using a constant particle diameter $d$ as specified in Table 1 and different values of pipe diameter $D$. We first investigate the total kinetic energy 

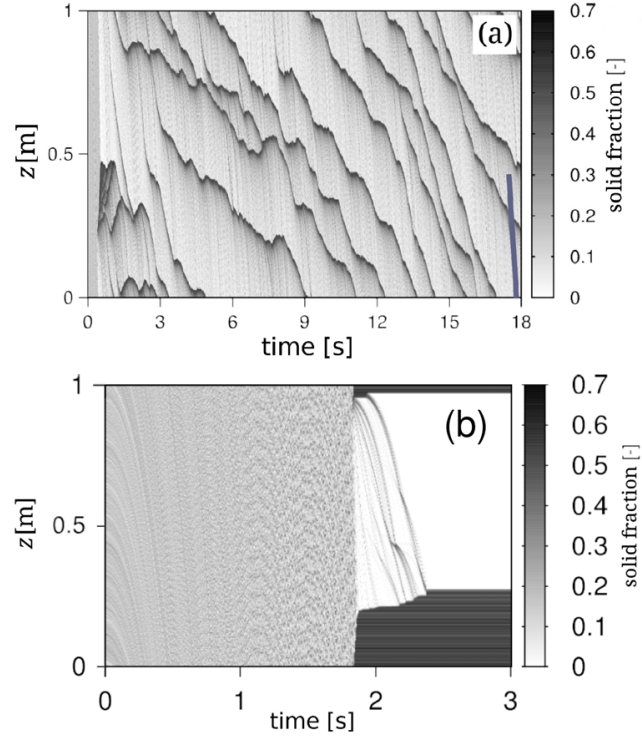

Figure 1. Spatio-temporal images of the packing fraction along the tube for a pipe to particle diameter $D / d=4$ (a) and $D / d=2.5$ (b). The slope of the line at the lower right corner in (a) indicates the average axial velocity of the particles.

of particles flowing through a vertical pipe with $D / d=4$. Collisions between the particles and between the particles and the wall lead, then, to deceleration of the particles and a decrease in the kinetic energy of the system. After a transient time, the energy gain of the particles due to gravity is nearly compensated by the energy dissipation due to collisions such that the total kinetic energy fluctuates around a constant value $[1,14]$.

The spatio-temporal image of the volume fraction along the tube (Fig. 1a) shows the emergence of density waves [1,14], with the development of recurrent clogging characterized by the formation of plugs, that is regions with high packing fraction (thick dark lines in Fig. 1a) which can either converge or diverge with time. The flow is inhomogeneous and associated with strong fluctuations of particle average velocities and solid fraction both in time and position along the pipe.

The average flow rate is dictated by the behavior of grains within the plug zones [19]. Each plug zone is associated with the formation of an unstable arch, the height of which should scale with $D$. Particles falling after the break of an arch can accelerate freely due to the action of gravity thus leading to a scaling of the average vertical velocity of the grains, $\left\langle v_{z}\right\rangle$, with $\sqrt{D}$ [19]. Therefore, a scaling of the average mass flux exiting the pipe through its bottom end per unit time, $Q_{0}$, with $D^{5 / 2}$ is expected. This scaling indeed governs the mass flux of a granular material flowing out a silo through an orifice of diameter $D$, which follows the well-known Beverloo equation [6, 9, 19, 20],

$$
Q_{0}=A \rho_{\mathrm{p}} \sqrt{g} \cdot(D-k d)^{2.5},
$$

where the coefficients $A$ and $k$ must be determined from the fit to the data. In Ref. [14] we have shown that such a
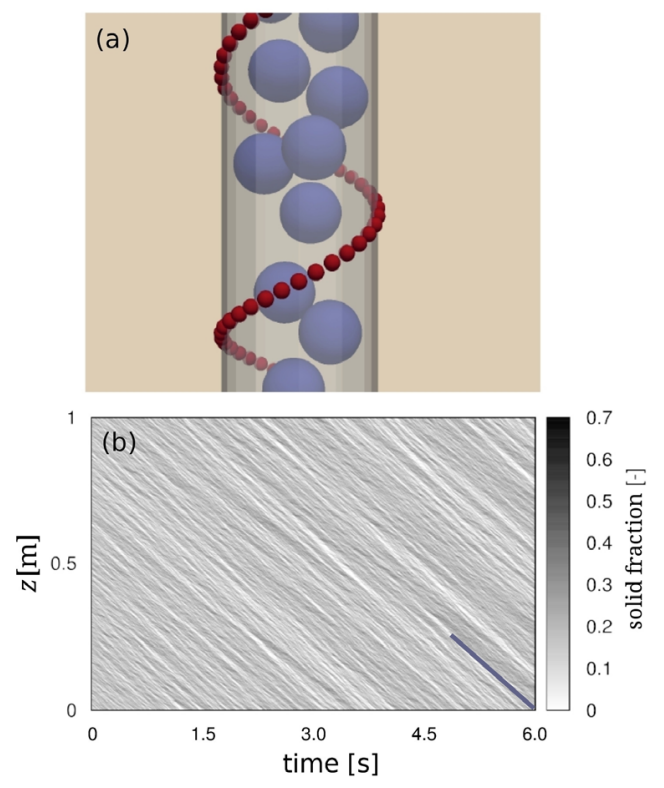

Figure 2. Flow of granular particles (blue) through a vertical pipe with $D / d=2.5$ to which the helical inner-wall texture (constituted by the red beads) is applied; (b) corresponding spatiotemporal diagram of the solid fraction along the axial position. The slope of the line at the lower right corner indicates the average axial velocity of the particles.

scaling captures well the dependence of the mass flux on the pipe diameter for $D / d>3$.

Moreover, we find that, for $D / d \lesssim 3$, jamming occurs thus leading to complete blockage of the granular flow. As an example of the flow in the jamming regime, we show in Fig. $1 \mathrm{~b}$ the spatio-temporal diagram of the packing fraction for $D / d=2.5$. As we can see in this figure, after about $1.8 \mathrm{~s}$, a large, stable plug is formed, which corresponds to the dark horizontal lines in the spatio-temporal diagram. Although we indeed did not observe complete flow blockage for $D / d>3.0$, the flow in this regime is intermittent and characterized by density waves as discussed in detail in Ref. [14] and demonstrated previously [1,2]. Therefore, in the following our aim is to develop a method to homogeneize the flow thus avoiding the formation of density waves that lead to jamming in granular pipe flows.

We investigate the vertical flow of a granular material down a pipe of circular cross-section that has a helical inner-wall texture as depicted in Fig. 2a. This texture is constituted of small beads of diameter $d_{s}=D / 10$, which are fixed to the inner-wall of the pipe and have the material properties listed in Table 1. Each constituent bead is fixed to the wall at its center such that one hemisphere of each bead is within the inner volume of the pipe. The diameter of the pipe in the presence of the helix is adjusted such that the total volume within the pipe is the same as in the simulations without the texture elements.

As we can see jamming does not occur in such a pipe in the presence of the helical texture. A steady downward flow of the granular material is observed, whereas the particles are more homogeneously distributed throughout the pipe compared to the simulations without the helical tex- 
ture. This result can be understood by noting that, as the particles collide with the beads fixed on the inner-wall, they are deflected to the center of the pipe, thus reducing the probability for the formation of stable archs. On the other hand, due to the collisions of the particles with the inner-wall beads, the average axial velocity of the particles is reduced, thus causing a decrease in the average mass flux in the axial direction [14]. Therefore, by introducing the helical texture of Fig. 2a, jamming can be prevented at the cost of a lower rate of particle flux down the pipe.

We find that the average mass flux depends significantly on the wavelength of the helix, $\lambda$. In order to quantitatively describe the effect of the helix wavelength on the mass flux, we first note that in the steady-state, the gain in momentum of the particles due to gravity is fully compensated by the momentum loss due to collisions with the pipe's inner-wall as well as with the other particles. In the presence of the helix, there is an additional contribution to the rate with which axial momentum is dissipated. This contribution, $\dot{p}_{\text {diss,helix }}$, is due to collisions of the particles with the beads composing the helix. It is reflected by a relative decrease in the magnitude of the steady-state axial velocity of the particles, compared to the value without helix [14]. Therefore, the steady-state flux $Q$ in the presence of the helix relates to the flux $Q_{0}$ without helix through the expression

$$
Q=Q_{0}-Q_{\text {diss,helix }},
$$

where $Q_{\text {diss,helix }}$ is the amount by which the steady-state axial mass flux is reduced when the helical texture is present.

In the small pipe to particle diameter ratio investigated here, it is reasonable to assume that the average mass flux in the axial direction is proportional to the average axial momentum of the particles, and that $Q_{\text {diss,helix }}$ is nearly proportional to $\dot{p}_{\text {diss, helix }}$. As a result of particle-helix collisions along the pipe, the particles are deflated to the pipe's central axis which is why there is an increase in the average radial momentum when the helix is present [14]. However, we note that due to symmetry, for any radial direction, the contribution of the collisions to increasing the radial momentum at the upper half of one helix wavelength is the same as at the lower half. For a given pipe diameter $D$ and an helix wavelength $\lambda$ made up of $n_{\mathrm{s}}$ beads, the collisions between particles with $n_{\mathrm{s}} / 2$ beads from the upper and lower hemispheres (half-wavelengths) deflate the particles in opposite directions. We thus need to calculate the rate with each the particles gain radial momentum due to collisions with one single hemisphere. The total rate is then twice the contribution from one half-hemisphere.

Fig. 3 represents both hemispheres unravelled from the inner-wall as two right triangles, each with legs $D$ and $\lambda / 2$. Note that $\lambda / 2$ is the length corresponding to $n_{\mathrm{s}} / 2$ beads, which is the contribution of each hemisphere to increase the radial momentum along the pipe's cross section. From the figure, we see that the rate of axial momentum dissipation due to each hemisphere must scale with $p_{\perp}$, the component of the particles' average axial momentum $p_{0}$ perpendicular to the hypothenuse of each triangle. Therefore, $\dot{p}_{\text {diss,helix }} \propto 2 p_{\perp}=2 p_{0} \sin \left[\Theta_{D, \lambda / 2}\right]$, where $\Theta_{D, \lambda / 2}=\arctan [\pi D /(\lambda / 2)]$ (see Fig. 3).

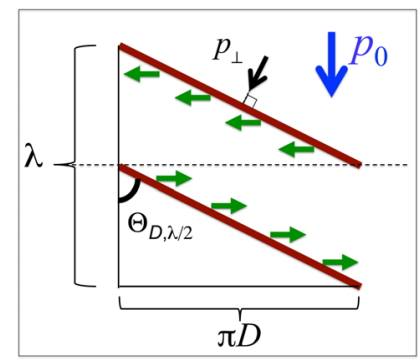

Figure 3. Schematic diagram displaying the average axial momentum $p_{0}$ and the equivalent angle $\Theta_{D, \lambda / 2} \equiv \arctan [\pi D /(\lambda / 2)]$, which dictates the energy dissipation over one helix wavelength due to collisions with the $n_{\mathrm{s}} / 2$ beads composing one half-wavelength of the helix. The component $p_{\perp}=$ $p_{0} \sin [\arctan [\pi D /(\lambda / 2)]]$ is also indicated. The horizontal arrows along the helix indicate the direction from the helix to the central axis of the pipe.

Following our assumption that $Q_{\text {diss,helix }}$ should be proportional to $\dot{p}_{\text {diss,helix }}$ we can write $Q_{\text {diss,helix }} \approx 2 b Q_{0} \sin [\arctan [\pi D /(\lambda / 2)]]=$ $2 b Q_{0} \cdot\left[2 \pi D / \sqrt{\lambda^{2}-(4 \pi D)^{2}}\right]$, where $b$ is a parameter that encodes information on the dissipative properties of the collisions. Thus, from Eq. (2),

$$
\frac{Q}{Q_{0}}=1-B\left[\frac{2 \pi D}{\sqrt{\lambda^{2}-(2 \pi D)^{2}}}\right],
$$

where $Q_{0}$ is the flux without helix and the constant $B=2 b$ encodes information on energy dissipation due to collisions with the helix. Thus, the value of $B$ should depend on the material properties and particle diameter. To verify Eq. (3), we compute the expectation value of the flux, $Q=\int q f_{\lambda}(q) d q$ as a function of $\lambda$, where $f_{\lambda}(q)$ is the probability density function associated with the wavelength $\lambda$. The result of this calculation is denoted by the symbols in Fig. 4b, in which $Q$ appears rescaled with $Q_{0}$. The continuous line denotes the best fit using Eq. (3), which gives $B \approx 0.74$. As we can see from this figure, the agreement between model and simulation results is excellent.

The inset of Fig. $4 \mathrm{~b}$ shows the rescaled standard deviation of the flux, $\sigma / \sigma_{0}$, as a function of $1 / \lambda$, where $\sigma_{0}$ is the standard deviation obtained in the simulations without helix. The value of $\sigma / \sigma_{0}$ gives a measure of the homogeneity of the flux along the pipe - the smaller $\sigma$ the more homogeneous the flux. By fitting the simulation data using the equation

$$
\frac{\sigma}{\sigma_{0}}=1-C \cdot\left[\frac{2 \pi D}{\sqrt{\lambda^{2}-(2 \pi D)^{2}}}\right],
$$

we obtain $C \approx 0.9$. In conclusion, both the flux and its standard deviation can be obtained from the geometric parameters of the helix using Eqs. (3) and (4), respectively.

However, obviously Eq. (3) can only be valid for the regime of small pipe to particle diameter ratios investigated here. In particular, it can be shown that the model predicts negative flux values for $D / d \gtrsim 10$ [14]. Moreover, 


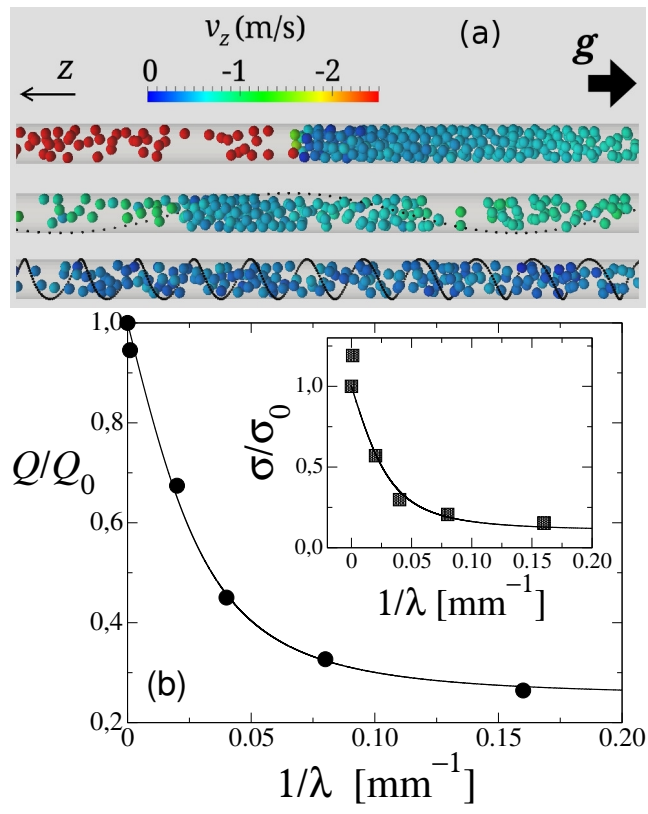

Figure 4. (a) Simulations with no helix and with helix of wavelength $\lambda=25 \mathrm{~mm}$ and $6.25 \mathrm{~mm}$ (from top to bottom); (b) expectation value of the rescaled mass flux $Q / Q_{0}$ (main plot) and non-dimensional standard deviation $\sigma / \sigma_{0}$ (inset) as a function of $1 / \lambda$. Symbols denote simulation results, continuous lines are best fits to the model described in the text, while $D / d=3.5$, $Q_{0} \approx 0.0074 \mathrm{~kg} / \mathrm{m}^{3}$ and $\sigma_{0} \approx 0.00025 \mathrm{~kg} / \mathrm{m}^{3}$.

as $\lambda \rightarrow 0$ the flux through the pipe must follow the original Beverloo equation with $Q_{0}$ corresponding to a pipe with no helix and diameter $D-d_{\mathrm{s}}$, where $d_{\mathrm{s}}$ is the size of the spheres constituting the helix. Further work is thus needed in order to elucidate the dependence of model fit parameters on the number of helix beads per wavelength $\lambda$, as well as on the particles' frictional and dissipation properties.

\section{Conclusions}

In conclusion, we have presented a method to obtain steady flows of granular materials through narrow pipes, which consists of applying a helical texture to the pipe's inner wall. The helix texture prevents the formation of stable plugs and avoids jamming of the granular flow. We believe that application of the helical texture presented here could be used to enhance not only gravity-driven pipe flows but also fluid-driven particle transport through both through vertical and horizontal pipes, which remains to be investigated in the future. Moreover, the influence of wear should be also investigated.

\section{Acknowledgements}

We thank the German Research Foundation (DFG) for funding through the Cluster of Excellence "Engineering of Advanced Materials", ZISC, FPS, the Collaborative Research Center SFB814, and grant PO472/20-2. We gratefully acknowledge the computing time granted by the John von Neumann Institute for Computing (NIC) and provided on the supercomputer JUROPA at Jülich Supercomputing Centre (JSC).

\section{References}

[1] T. Pöschel, J. Phys. I France 4, 499-506 (1994).

[2] T. Riethmüller, L. Schimansky-Geier, D. Rosenkranz and T. Pöschel, J. Stat. Phys. 86, 421-430 (1997).

[3] J. L. Aider, N. Sommier, T. Raafat and J. P. Hulin, Phys. Rev. E 59, 778-786 (1999).

[4] Y. Bertho, F. Giorgiutti-Dauphiné, T. Raafat, E. J. Hinch, H. J. Herrmann and J. P. Hulin, J. Fluid Mech. 459, 317-345 (2002).

[5] I. Bratberg, F. Radjai and A. Hansen, Phys. Rev. E 71, 011301 (2005).

[6] A. Janda, I. Zuriguel, J. Bienzobas, A. Garcimartín and D. Maza, AIP Conf. Proc. 1542, 710 (2013).

[7] W. Chen, M. Hou, K. Lu, Z. Jiang and L. Lam, Phys. Rev. E 64, 061305 (2001).

[8] M. L. Hunt, R. C. Weathers, A. T. Lee, C. E. Brennen and C. R. Wassgren, Physics of Fluids 11, 68-75 (1999).

[9] I. Zuriguel, A. Janda, A. Garcimartín, C. Lozano, R. Arévalo and D. Maza, Phys. Rev. Lett. 107, 278001 (2011).

[10] N. V. Brilliantov, F Spahn, J. M. Hertzsch, T. Pöschel, Physical review E 53, 5382 (1996).

[11] T. Pöschel and T. Schwager, Computational Granular Dynamics (Springer, Heidelberg, 2005).

[12] P.A. Cundall, O.D.L. Strack, Geotechnique 29, 47 (1979).

[13] C. Kloss, C. Goniva, A. Hager, S. Amberger and S. Pirker, Prog. Comput. Fluid Dy. 12, 264 (2012). www. liggghts.com

[14] F. Verbücheln, E. J. R. Parteli and T. Pöschel, Soft Matter 11, 4295-4305 (2015).

[15] E. J. R. Parteli, AIP Conf. Proc. 1542, 185-188 (2013).

[16] H. Pourtavakoli, E. J. R. Parteli and T. Pöschel, New J. Phys. 18, 073048 (2016).

[17] E. J. R. Parteli and T. Pöschel, Powder Technology 288, 96-102 (2016).

[18] E. J. R. Parteli, J. Schmidt, C. Blümel, K.-E. Wirth, W. Peukert and T. Pöschel, Scientific Reports 4, 6227 (2014).

[19] C. Mankoc, A. Janda, R. Arévalo, J. M. Pastor, I. Zuriguel, A. Garcimartín and D. Maza, Granular Matter 9, 407 (2007).

[20] W. A. Beverloo, H. A. Leniger and J. van de Velde, Chemical Engineering Science 15, 260-269 (1961). 